\title{
Overestimation of Mean Heart Rate by the Arithmetic Average of Beat- By-Beat Sampled Heart Rate Values
}

\author{
Harald M. Stauss* and Kevin R. Rarick \\ Department of Health and Human Physiology, The University of Iowa, 410 Field House, Iowa City, IA 52242, USA
}

\begin{abstract}
The arithmetic average of beat-by-beat sampled heart rate (HR) values overestimates true HR defined as number of heart beats per time unit. The aims of this study were to (1) estimate the magnitude of overestimation; (2) illustrate the significance of this issue using data from patients with congestive heart failure (CHF) and control subjects; and (3) outline approaches to correctly calculate mean HR.

Linear regression analysis of computer-generated time series, representing beat-by-beat HR values in humans, rats, and mice, revealed that the difference between the arithmetic average of beat-by-beat sampled HR values and the true mean HR (error $\varepsilon$ ) can be approximated by the variance $\left(\sigma^{2}\right)$ divided by the arithmetic average $(\mu)$ of the beat-by-beat HR values $\left(\varepsilon=\sigma^{2} / \mu\right)$.

True mean HR was higher in patients with CHF $(92.9 \pm 4.3 \mathrm{bpm})$ than in control subjects $(82.6 \pm 2.1 \mathrm{bpm}, \mathrm{P}=0.045)$. However, if mean HR was calculated as arithmetic average of the beat-by-beat HR values the difference in mean $H R$ was no longer significant (93.4 $\pm 4.4 \mathrm{bpm}$ in CHF vs. 83.8 $\pm 2.1 \mathrm{bpm}$ in controls, $\mathrm{P}=0.059$ ).

In conclusion, the arithmetic average of beat-by-beat sampled HR values overestimates true HR by approximately the ratio of $\sigma^{2}$ to $\mu$ of the beat-by-beat HR values. Thus, the error $(\varepsilon)$ is largest in subjects with high HR variability and low average HR and may affect interpretation of mean HR values in studies investigating populations of subjects with differing HR variability, such as CHF patients vs. healthy subject or old vs. young subjects.
\end{abstract}

Keywords: Computation, heart rate variability, hemodynamic analysis, pulse interval, RR interval, time series analysis.

\section{INTRODUCTION}

The importance of heart rate (HR) as a cardiovascular parameter is evident by considering the reduced resting HR in endurance trained athletes [1], the increased resting HR in patients with congestive heart failure [2], or the negative prognostic value of low HR variability [3]. Various data acquisition systems and data analysis software packages allow investigators to generate spreadsheets with highly accurate beat-by-beat sampled HR values derived from ECG or blood pressure (BP) waveform recordings. The easiest way to calculate mean HR from such beat-by-beat sampled HR values is to use the arithmetic average function implemented in most spreadsheet software (e.g., Microsoft Excel). However, investigators may not be aware of the problem that the arithmetic average of beat-by-beat sampled HR values is greater than the true HR calculated as the number of heart beats divided by the recording duration [46]. This problem is illustrated in Fig. (1) that shows HR during pacing at $60 \mathrm{bpm}$ for $5 \mathrm{~min}$ followed by another $5 \mathrm{~min}$ of pacing at $100 \mathrm{bpm}$. The arithmetic average of the beat-by-beat sampled HR values is: 300 beats at $60 \mathrm{bpm}$ plus 500 beats at $100 \mathrm{bpm}=(300 * 60 \mathrm{bpm}+500 * 100 \mathrm{bpm}) / 800$ beats $=85 \mathrm{bpm}$. However, per definition, the true mean HR

*Address correspondence to this author at the The University of Iowa, Department of Health and Human Physiology, 410 Field House, Iowa City, IA 52242, USA; Tel: +1-319-335-9494; Fax: +1-319-335-6966; E-mail: harald-stauss@uiowa.edu should be calculated as the number of beats per time, which equates to 800 beats in $10 \mathrm{~min}$ or $80 \mathrm{bpm}$. Thus, in this example, the arithmetic average overestimates true mean HR by $5 \mathrm{bpm}$.

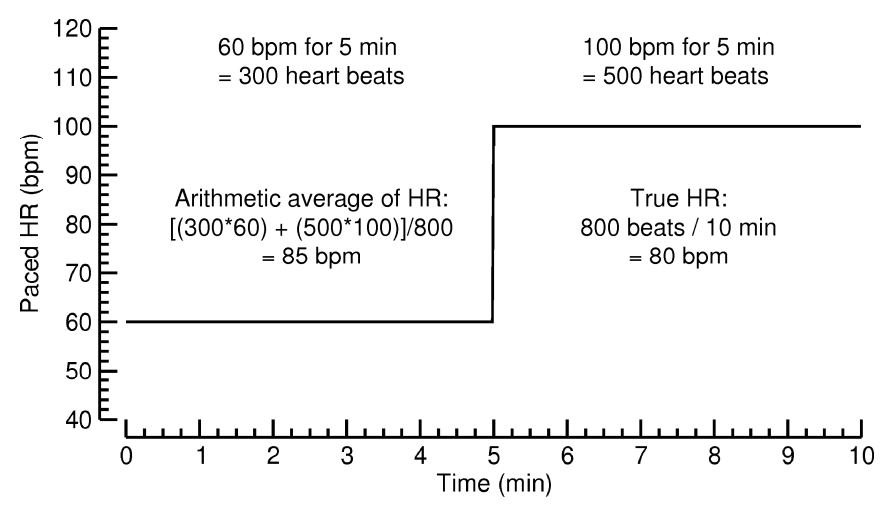

Fig. (1). Illustration of the error made by calculating mean heart rate (HR) by the arithmetic average of beat-by-beat sampled HR values.

Based on these considerations, the objectives of this article are: (1) to estimate the magnitude of the error made by incorrectly calculating mean HR as the arithmetic average of beat-by-beat sampled HR values; (2) to use real world ECG data from patients with congestive heart failure and healthy control subjects to test if the error made by calculating mean $\mathrm{HR}$ as the arithmetic average is large 
enough to affect interpretation of mean HR values; and (3) to outline approaches to correctly calculate mean HR.

\section{METHODS}

\section{Error Estimation by Computer Simulations}

To estimate the error $\varepsilon$ made by incorrectly calculating mean HR as the arithmetic average of beat-by-beat sampled HR values, we generated 1000 evenly distributed random numbers between 0 and 1 using the "RAND" function of the Microsoft Excel spreadsheet software. We then applied a zscore-based transform to these random numbers to generate 1) 10 time series with an average of 70 and variances of 20 , 40, 60, 80, 100, 120, 140, 160, 180, 200 (representing HR time series in humans); 2) 10 time series with an average of 300 and variances of 100, 200, 300, 400, 500, 600, 700, 800, 900, 1000 (representing HR time series in rats), and 3) 10 time series with an average of 500 and variances of 200, 400, $600,800,1000,1200,1400,1600,1800,2000$ (representing HR time series in mice). From these 30 computer-generated HR time series mean HR was calculated incorrectly as the arithmetic average and correctly as the number of heart beats (1000) divided by the recording time (sum of the inverse of the individual HR values). The error $\varepsilon$ was then calculated as the difference between these two mean HR values. Finally, we investigated the relationship between the variability of the HR time series and the error $\varepsilon$ by linear regression analysis between the variances $\sigma^{2}$ of the HR time series and the error values $\varepsilon$.

\section{Error Estimation Using Real World Data from Patients with Congestive Heart Failure}

\section{Subjects}

ECG recordings from 15 subjects (11 men, age 22-71 and 4 women, age 54-63) with severe congestive heart failure (NYHA class 3-4) were obtained from the PhysioBank database of PhysioNet (http://www.physionet.org/) [7]. This group of subjects was part of a larger study group receiving conventional medical therapy prior to receiving the oral inotropic agent, milrinone [8]. The individual recordings are each about 20 hours in duration, and contain two ECG signals, each sampled at 250 samples per second with 12-bit resolution over a range of \pm 10 millivolts. Additional longterm ECG recordings from 18 subjects (5 men, age 26-45 and 13 women, age 20-50) were also obtained from the same database. These subjects had been referred to the Arrhythmia Laboratory at Boston's Beth Israel Hospital (now the Beth Israel Deaconess Medical Center) but were found to have had no significant arrhythmias. For the purpose of this study we consider these subjects as healthy controls.

\section{Data Analysis}

The PhysioBank database also provided annotation files that were prepared using an automated detector. These annotation files were used to directly retrieve the beat-bybeat RR intervals and the corresponding time of the day from all ECG recordings. From these RR-interval time series, 5 hours of daytime recording were extracted starting at 2:00 PM and ending at 7:00 PM. These 5-hour RR-interval time series were then visualized on the computer screen and
RR-intervals that apparently (based on visual inspection) originated from cardiac arrhythmias such as extrasystoles were replaced by interpolated values using the HemoLab software (www.haraldstauss.com/HemoLab/HemoLab.html). It turned out that one recording from a patient with heart failure (subject no. 2) needed to be excluded because of too many cardiac arrhythmias. Thus, 14 patients with congestive heart failure and 18 control subjects were included in the study. The final 5-hour RR-interval time series were converted to beat-by-beat HR time series by the inverse of each RR-interval value (in s) multiplied by 60 . From these HR time series, mean HR was calculated incorrectly as the arithmetic average and correctly by the number of HR values divided by the recording time (sum of all RR-interval values in minutes). Finally, the error $\varepsilon$ was calculated as the difference between these two mean HR values and the variance $\sigma^{2}$ of all HR time series was calculated for linear regression analysis between the variance $\sigma^{2}$ and the error $\varepsilon$.

\section{Statistics}

All data are presented as means \pm SEM. Comparisons between parameters in patients with congestive heart failure and healthy control subjects were performed using unpaired Student t-tests. Statistical significance was assumed for $\mathrm{P}<0.05$.

\section{RESULTS}

\section{Error Estimation by Computer Simulations}

We applied linear regression analyses to computergenerated time series representing beat-by-beat HR values in humans, rats, and mice (Fig. 2). These analyses revealed three important findings: First, the error $\varepsilon$ is always positive, demonstrating that calculating mean $\mathrm{HR}$ as the arithmetic average overestimates true HR. Second, the correlation between the variance $\sigma^{2}$ and the error $\varepsilon$ made by incorrectly calculating mean $\mathrm{HR}$ as the arithmetic average revealed perfectly linear relationships $(\mathrm{R}=1.0)$. Third, the slopes of the linear regression lines were close to the inverse of the arithmetic average $(\mu)$ of the computer-generated data files

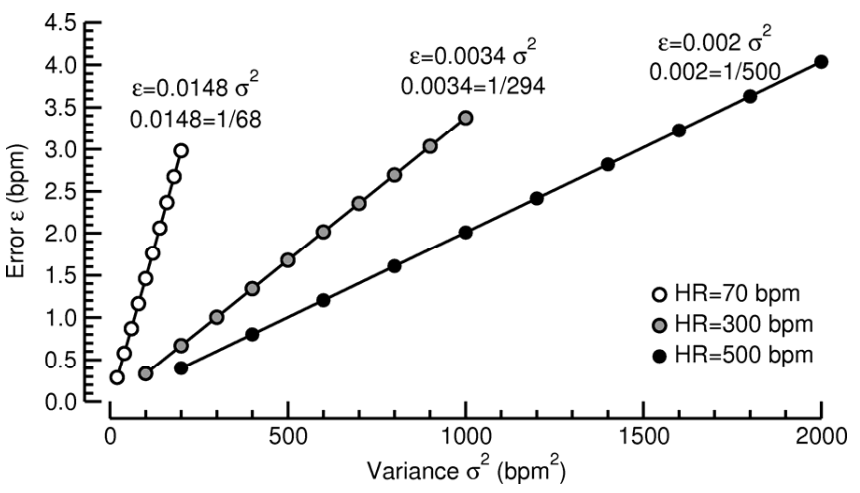

Fig. (2). Correlation between the variance $\sigma^{2}$ of beat-by-beat sampled heart rate (HR) values and the error $\varepsilon$ made by incorrectly calculating $\mathrm{HR}$ as the arithmetic average of beat-by-beat sampled HR values (y-axis). Computer-generated random data sets simulating HR time series in humans (open circles), rats (grey circles) and mice (black circles) are shown. 
representing beat-by-beat $\mathrm{HR}$ time series from humans (70 bpm, 1/slope $=68)$, rats $(300 \mathrm{bpm}, 1 / \mathrm{slope}=294)$, and mice $(500 \mathrm{bpm}, 1 / \mathrm{slope}=500)$. These findings indicate that the error $\varepsilon$ can be approximated by the variance of the HR time series divided by the arithmetic average $(\mu)$ of the HR time series $\left(\varepsilon=\sigma^{2} / \mu\right)$.

\section{Error Estimation Using Real World Data from Patients with Congestive Heart Failure}

Five hours of daytime beat-by-beat HR time series from 14 patients with congestive heart failure and 18 healthy control subjects were analyzed. Mean HR (correctly calculated) was $92.9 \pm 4.3 \mathrm{bpm}$ and $82.6 \pm 2.1 \mathrm{bpm}$ in patients and controls, respectively. This difference in mean $\mathrm{HR}$ was statistically significant $(\mathrm{P}=0.045)$. HR variability expressed as variance $\sigma^{2}$ of the beat-by-beat $H R$ values was $59 \pm 21$ $\mathrm{bpm}^{2}$ and $102 \pm 10 \mathrm{bpm}^{2}$ in patients and controls, respectively. This trend towards reduced HR variability in patients with congestive heart failure was not statistically significant $(\mathrm{P}=0.07)$. The error $\varepsilon$ made by calculating mean HR incorrectly as the arithmetic average ranged from $0.066 \mathrm{bpm}$ to 2.339 bpm (Fig. 3) and was significantly $(\mathrm{P}<0.05)$ smaller in patients with congestive heart failure $(0.58 \pm 0.18 \mathrm{bpm})$ than in control subjects $(1.19 \pm 0.11 \mathrm{bpm})$. Thus, mean HR calculated incorrectly as the arithmetic average was overestimated more in control subjects than in patients with congestive heart failure. This greater overestimation of mean HR in control subjects resulted in there no longer being a statistically significant difference between the two groups $(83.8 \pm 2.1$ bpm vs. 93.5 $\pm 4.4 \mathrm{bpm}$ controls vs. patients, $\mathrm{P}=0.059)$ when mean HR is calculated incorrectly as the arithmetic average.

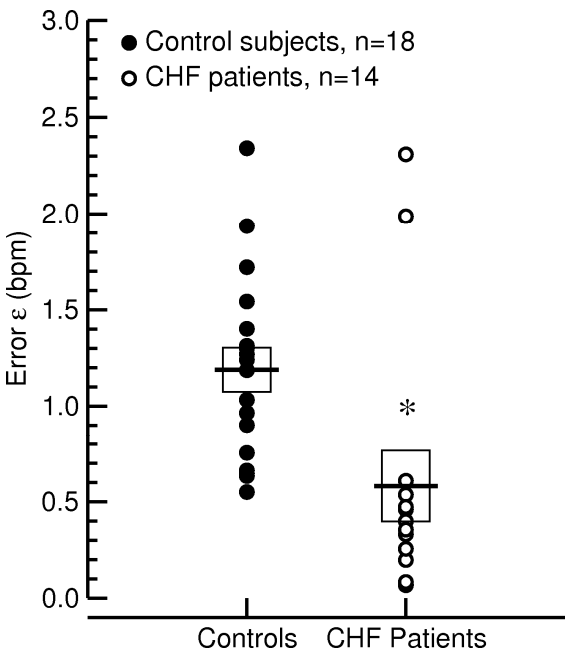

Fig. (3). Error $\varepsilon$ made by incorrectly calculating heart rate (HR) as the arithmetic average of beat-by-beat sampled HR values in patients with congestive heart failure (CHF, white circles) and healthy control subjects (black circles). The horizontal lines with the boxes around them indicate the means \pm SEM. *: $\mathrm{P}<0.05$ vs. Controls.

To test if the equation $\varepsilon=\sigma^{2} / \mu$, determined by computersimulations holds true for "real world data", we plotted the error values $\varepsilon$ versus the HR variability expressed as variance $\sigma^{2}$ for all 32 subjects (Fig. 4) and determined the correlation coefficient $\mathrm{R}^{2}$ for the regression line determined by the equation $\varepsilon=\sigma^{2} / \mu$ with $\mu$ defined as the average HR of all 32 subjects $(88.0 \pm 2.4 \mathrm{bpm}$, patients and control subjects pooled). Thus, the regression line shown in Fig. (4) is not the "best fit" or "trendline" through the data points, but simply the line represented by the equation $\varepsilon=(1 / 88) * \sigma^{2}$. The correlation coefficient $\mathrm{R}^{2}$ of 0.93 , strongly suggests that the equation $\varepsilon=\sigma^{2} / \mu$ is an acceptable approximation of the true error $\varepsilon$ not only for computer-generated but also for "real world" data.

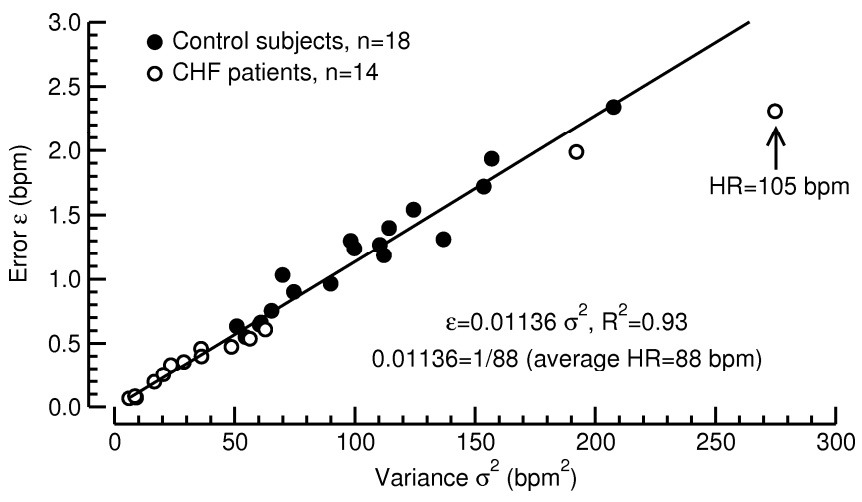

Fig. (4). Correlation between the variance $\sigma^{2}$ and the error $\varepsilon$ made by incorrectly calculating HR as the arithmetic average of beat-bybeat sampled HR values in patients with congestive heart failure (CHF, white circles, $n=14$ ) and healthy control subjects (black circles, $\mathrm{n}=18$ ). The regression line was determined by the equation $\varepsilon=\sigma^{2} / \mu$ with $\mu$ defined as the average HR of all 32 subjects (patients and control subjects pooled). The values for the patient at $\sigma^{2}=275 \mathrm{bpm}^{2}$ and $\varepsilon=2.3 \mathrm{bpm}$ is somewhat off the regression line, because the $\mathrm{HR}$ in this patient was $105 \mathrm{bpm}$ and deviates substantially from the average HR of all patients ( $88 \mathrm{bpm})$.

\section{DISCUSSION}

There are two major new findings from this study. First, the arithmetic average of beat-by-beat sampled HR values overestimates true mean HR by an amount $\varepsilon$ that can be approximated by the ratio of the variance $\sigma^{2}$ and the arithmetic average $\mu$ of the HR values $\left(\varepsilon=\sigma^{2} / \mu\right)$. Second, from all 32 subjects included in this study the largest error $\varepsilon$ made by incorrectly calculating mean HR as the arithmetic average was $2.3 \mathrm{bpm}$ (Fig. 3). Thus, the error $\varepsilon$ appears to be relatively small and interpretation of mean HR values may not be affected by this error in a majority of studies.

The importance of the equation $\varepsilon=\sigma^{2} / \mu$ is that it allows identification of studies that are most likely to be affected by the error $\varepsilon$. According to this equation, the error is largest if HR variability is high and mean HR is low. Thus, studies in subjects with differing HR variability and/or mean HR are most prone to misinterpretation of mean $\mathrm{HR}$ values. Examples include studies involving endurance trained athletes, in which HR variability is high and mean HR is low (1) or heart failure patients, in which HR variability is low and mean HR is high (2). In endurance trained athletes the low HR would be overestimated more, while in heart failure patients the high HR would be overestimated less than in control subjects. Thus, in both populations of subjects a true difference in mean HR compared to control subjects may be missed if mean HR is incorrectly calculated as the arithmetic 
average of beat-by-beat sampled HR values. Indeed, in our study, the error $\varepsilon$ was found to be significantly smaller in patients with congestive heart failure than in healthy control subjects (Fig. 3). Furthermore, the difference in mean HR in the two groups of subjects was significant if $\mathrm{HR}$ was calculated correctly $(\mathrm{P}=0.045)$ but missed the level of significance if mean HR was calculated incorrectly as the arithmetic average $(\mathrm{P}=0.059)$.

Mean HR can be calculated correctly from beat-by-beat sampled HR values by several methods. One approach is to first convert the beat-by-beat sampled HR values in RR intervals (if derived from ECG) or pulse intervals (if derived from arterial blood pressure waveforms) by calculating the inverse $(1 / x)$ for each beat-by-beat HR value. Then the arithmetic average of the beat-by-beat RR interval or pulse interval values can be calculated. Finally, the average RR interval or pulse interval can be converted back to mean HR by taking the inverse $(1 / \mathrm{x})$ of the average $\mathrm{RR}$ interval or pulse interval. A second method is to simply divide the number of heart beats by the recording duration (in minutes). If the recording duration is not known, it can be calculated as the sum of the inverse $(1 / x)$ of the beat-by-beat HR values. To test if a given data analysis software calculates HR correctly, investigators may compare the software-calculated mean HR and mean RR interval or mean pulse interval values for consistent results. If mean HR turns out to be higher than the inverse of the mean RR interval or mean pulse interval, it is likely that mean HR was incorrectly calculated as the arithmetic average of the beat-by-beat sampled HR values.

In conclusion, the arithmetic average of beat-by-beat sampled HR values overestimates true mean HR by an amount of approximately the variance divided by the arithmetic average of the beat-by-beat HR values. This overestimation is generally small and may only be relevant in studies involving populations of subjects with differing HR variability and/or mean HR values.

\section{ACKNOWLEDGEMENT}

None declared.

\section{CONFLICT OF INTEREST}

None declared.

$\begin{array}{ll}\text { ABBREVIATIONS } \\ \mathrm{BP} & =\text { Blood Pressure } \\ \mathrm{bpm} & =\text { Beats per minute } \\ \mathrm{CHF} & =\text { Error } \\ \varepsilon & =\text { Electrocardiogram } \\ \mathrm{ECG} & =\text { Heart Rate } \\ \mathrm{HR} & =\text { Arithmetic Average } \\ \mu & =\text { Pew York Heart Association } \\ \mathrm{NYHA} & =\text { Correlation Coefficient } \\ \mathrm{P} & =\text { Time interval from one R-wave to the next } \\ \mathrm{R} & =\text { R-wave } \\ \text { RR- } & =\text { Standard Error of the Mean } \\ \text { interval } & \end{array}$

\section{REFERENCES}

[1] Cornolo J, Brugniaux JV, Macarlupu JL, Privat C, Leon-Velarde F, Richalet JP. Autonomic adaptations in Andean trained participants to a 4220-m altitude marathon. Med Sci Sports Exerc 2005; 37 (12): 2148-53.

[2] Van Hoogenhuyze D, Weinstein N, Martin GJ, et al. Reproducibility and relation to mean heart rate of heart rate variability in normal subjects and in patients with congestive heart failure secondary to coronary artery disease. Am J Cardiol 1991; 68: 1668-76.

[3] Kleiger RE, Miller JP, Bigger JT Jr, Moss AJ. Decreased heart rate variability and its association with increased mortality after acute myocardial infarction. Am J Cardiol 1987; 59(4): 256-62.

[4] Thorne PR, Engel BT, Holmblad JB. An analysis of the error inherent in estimating heart rate from cardiotachometer records. Psychophysiology 1976; 13(3): 269-72.

[5] Jennings JR, Berg WK, Hutcheson JS, Obrist P, Porges S, Turpin G. Committee report. Publication guidelines for heart rate studies in man. Psychophysiology 1981; 18(3): 226-31.

[6] Reyes del Paso GA, Vila J. The continuing problem of incorrect heart rate estimation in psychophysiological studies: an off-line solution for cardiotachometer users. Biol Psychol 1998; 48(3):26979 .

[7] Goldberger AL, Amaral LA, Glass L, et al. PhysioBank, PhysioToolkit, and PhysioNet: components of a new research resource for complex physiologic signals. Circulation 2000; 101(23): E215-20.

[8] Baim DS, Colucci WS, Monrad ES, et al. Survival of patients with severe congestive heart failure treated with oral milrinone. J Am Coll Cardiol 1986; 7(3): 661-70. 